Article

\title{
On the Travel Emissions of Sustainability Science Research
}

\author{
Timothy Waring *, Mario Teisl ${ }^{\dagger}$, Eva Manandhar ${ }^{\dagger}$ and Mark Anderson ${ }^{\dagger}$ \\ Sustainable Solutions Initiative, School of Economics, University of Maine, Orono, ME 04469, USA; \\ E-Mails: teisl@maine.edu (M.T.); eva.manandhar@maine.edu (E.M.); mark.anderson@maine.edu (M.A.) \\ $\dagger$ These authors contributed equally to this work. \\ * Author to whom correspondence should be addressed; E-Mail: timothy.waring@maine.edu; \\ Tel.: +1-207-581-3157; Fax: +1-207-581-4278.
}

Received: 19 February 2014; in revised form: 17 April 2014 / Accepted: 18 April 2014 / Published: 8 May 2014

\begin{abstract}
This paper presents data on carbon emissions generated by travel undertaken for a major sustainability science research effort. Previous research has estimated $\mathrm{CO}_{2}$ emissions generated by individual scientists, by entire academic institutions, or by international climate conferences. Here, we sought to investigate the size, distribution and factors affecting the carbon emissions of travel for sustainability research in particular. Reported airline and automobile travel of participants in Maine's Sustainability Solutions Initiative were used to calculate the carbon dioxide emissions attributable to research-related travel over a three-year period. Carbon emissions varied substantially by researcher and by purpose of travel. Travel for the purpose of dissemination created the largest carbon footprint. This result suggests that alternative networking and dissemination models are needed to replace the high carbon costs of annual society meetings. This research adds to literature that questions whether the cultural demands of contemporary academic careers are compatible with climate stabilization. We argue that precise record keeping and routine analysis of travel data are necessary to track and reduce the climate impacts of sustainability research. We summarize the barriers to behavioral change at individual and organizational levels and conclude with suggestions for reducing climate impacts of travel undertaken for sustainability research.
\end{abstract}

Keywords: carbon footprint; sustainability science; travel; research culture; climate change; university carbon emissions 


\section{Introduction}

Achieving climate stabilization requires significant reductions in anthropogenic carbon emissions [1]. This requires an accurate understanding of the climate system, and the role of human alterations to that system, so that efforts to stabilize the climate are effective, and avoid unforeseen consequences [2-4]. It also requires an understanding of the nature of behavioral, social and institutional change so that policies and incentives have the greatest possible effect and highest efficiency [5,6]. The same sorts of knowledge are also required to approach any issue in sustainability science generally [7]. However, conducting research and disseminating results requires travel, and travel adds to atmospheric $\mathrm{CO}_{2}$ concentrations directly. Thus, for scientists involved in research and advocacy around issues of climate change, and environmental sustainability more generally, there is a tradeoff between the value of their research and dissemination activities and costs of the greenhouse gases emitted in the course of those activities.

To people outside the research sector, the environmental costs of researcher travel can appear hypocritical. For instance, one widely circulated political cartoon depicts a small private jet in the air, $\mathrm{CO}_{2}$ spewing from the engines, and two speech bubbles: "Well, Mr. Gore, are you on the way to another one of your seminars?" "Actually, I'm picking up a Nobel Prize for encouraging reduction of carbon emissions" [8]. To make matters worse, the perceived inauthenticity of scientists and advocates is likely to add legitimacy to the view that climate change is either not real or not serious, and further retard the progress of policies meant to address climate change. This presents the climate and sustainability research community with a complex but important dilemma: how to balance the tradeoff between the research enterprise and its environmental and social costs?

Accurate information about these costs and benefits can help to balance that tradeoff and inform debate, but depend on accurate accounting. Accounting and reporting standards for greenhouse gas emissions now exist for corporations, cities, product life cycle analyses, as well as many sector specific emissions calculation tools $[9,10]$. The International Standards Organization has adopted standard ISO 14064-1 on "Specification with guidance at the organization level for quantification and reporting of greenhouse gas emissions and removals." Based on these international standards, the Higher Education Funding Council for England's (HEFCE) has introduced a carbon reductions strategy and reporting framework for higher education [11]. Similar standardized approaches exist in the United States [12-14] although they remain voluntary.

There is a growing and important literature around the issue of travel emissions incurred as part of climate and sustainability research programs. Michaelowa and Luhmkuhl [15] estimated the greenhouse gas emissions of international climate negotiations between 1991 and 2004 and suggested that an expenditure of an additional half million U.S. dollars could have made the whole process "greenhouse-gas neutral." Gremillet [16] suggested that "flying to meetings to protect the environment" was a paradox and asked "whether the carbon footprints of ecologists outweigh the environmental benefits of their findings and their lobbying." Some conservation biologists [17] found that their own estimated annual carbon footprint were more than double the American average, nearly ten times that of the global average. Most of this difference was attributable to airline travel related to professional obligations [17]. If professional travel for sustainability research constitutes a large 
fraction of the additional carbon emissions incurred by researchers, then travel patterns require close inspection, and are worthy of study in their own right.

There are numerous alternative policy responses to the problem of environmentally costly travel, but very little consensus on how to approach a common solution. Bossdorf et al. [18] argued that the response to this problem should be to make ecology conferences "carbon neutral" by purchasing carbon offsets. Anderson [19] suggests a different response, that "slow travel" by train should be used to replace air travel for scientists to attend conferences and other scholarly activities. This has sparked a debate around the issue of air travel and climate science [20,21], raising the provoking question: Is the travel undertaken for climate and sustainability research worth the costs it imposes on the environment via added greenhouse gas emissions?

The issue the greenhouse gas emissions generated by research-related travel by sustainability and climate change researchers is rarely addressed in the climate and sustainability research community, and is little cited in the climate change mitigation or adaptation literature. There is a need for both more data on the carbon costs of research travel, and for enhanced discussion of the tradeoffs involved. Our study responds to these needs by exploring the carbon emissions generated by research travel in a major sustainability science research project.

\section{Research Design and Methodology}

The environmental effects of research travel can be measured in multiple ways - the individual researcher [17], a research institution [22], a specific conference [18], or a multi-year process [15]. The focus of this research was the carbon emissions of research travel of individual university faculty members and graduate students generated as part of one large sustainability science research project. As such, the study was smaller in scale than institution-wide carbon footprinting like that of Ozawa-Meida et al. [22] undertaken in the UK. Our study focused on the individual researcher because he or she exercises more autonomy over travel decisions, and therefore over this one source of carbon emissions from the university enterprise.

Maine's Sustainability Solutions Initiative (SSI) was a five-year, \$20 million (US) project funded by the U.S. National Science Foundation through an Experimental Program to Stimulate Competitive Research (EPSCoR) grant to the University of Maine. The project included a broad portfolio of solutions-oriented sustainability issues relevant to the state of Maine, and included climate change as a focal area. We estimate the $\mathrm{CO}_{2}$ emissions of the research travel undertaken by faculty members and graduate students and funded by this project over its first three fiscal years, from 1 July 2009 to June 2012. Travel for the administration of research projects within the SSI were included, but travel for the administration of the project itself was not included in this study because some of this travel was funded through accounts outside of the specific project. Approximately $1.2 \%$ of the total NSF project budget was spent on travel (see Appendix).

We estimated emissions of individual researchers generated directly for research within this project. We did not measure travel emissions generated by other research projects, by teaching activities, and by personal travel. We chose the individual researcher as the unit of analysis to place research travel in the context of overall individual carbon emissions and to explore the culture of academic research as a part of the larger climate change issue. Our data constitutes an estimate of Scope 3 carbon emissions 
for research travel estimate for individual researchers [9]. The Higher Education Funding Council for England's (HEFCE) definition of Scope 3 emissions as "indirect emissions that organizations produce through their activities, but occur from sources not owned or controlled by the organization" $[11,22]$. We were not able to collect data on the full-time equivalent (FTE) or researcher effort dedicated to this project nor do we have data on researcher travel patterns beyond this project.

We used travel expense vouchers filed by project participants for the first three years of the project to construct a complete database of travel on this project. University regulations dictate that travel reimbursement documents include data on mileage driven for automobile trips and airport legs for air travel. We collected vehicle miles driven and airport legs flown for each trip taken, by individual, by trip. In total there were 407 different trips between 9 September 2009 and 2 December 2012. We coded each trip by travel leg, such that a single trip might include multiple airplane flights and car drives. There were a total of 1029 individual travel legs. All flights were economy class, per National Science Foundation regulations. Only 10 of the 407 trips included both car and airplane travel. Of the 407 trips documented on travel vouchers, 28 trips included recorded air travel legs for which no corresponding connecting flight could be found. Most of these were flights from Bangor, Maine to US cities such as Baltimore (BWI), Madison (MSN) or Denver (DEN). This likely occurred due to the route changes of air carriers, or to travelers omitting the connecting airports. For these cases we added or substituted New York's LaGuardia airport (LGA) into the travel itinerary to create a complete (and feasible) flight plan. Names of individuals were purged from the database prior to analysis, but individuals were first coded by academic rank and discipline type (social or bio-physical scientist). Figure 1 presents the geographical distribution of air travel in the continental United States. Using these data we were able to accurately estimate carbon dioxide emissions on a person trip basis.

Figure 1. Domestic travels by frequency in the continental United States, not including trips to Fairbanks, AK (1), Honolulu, HI (2), and Edmonton, Alberta, Canada (1).

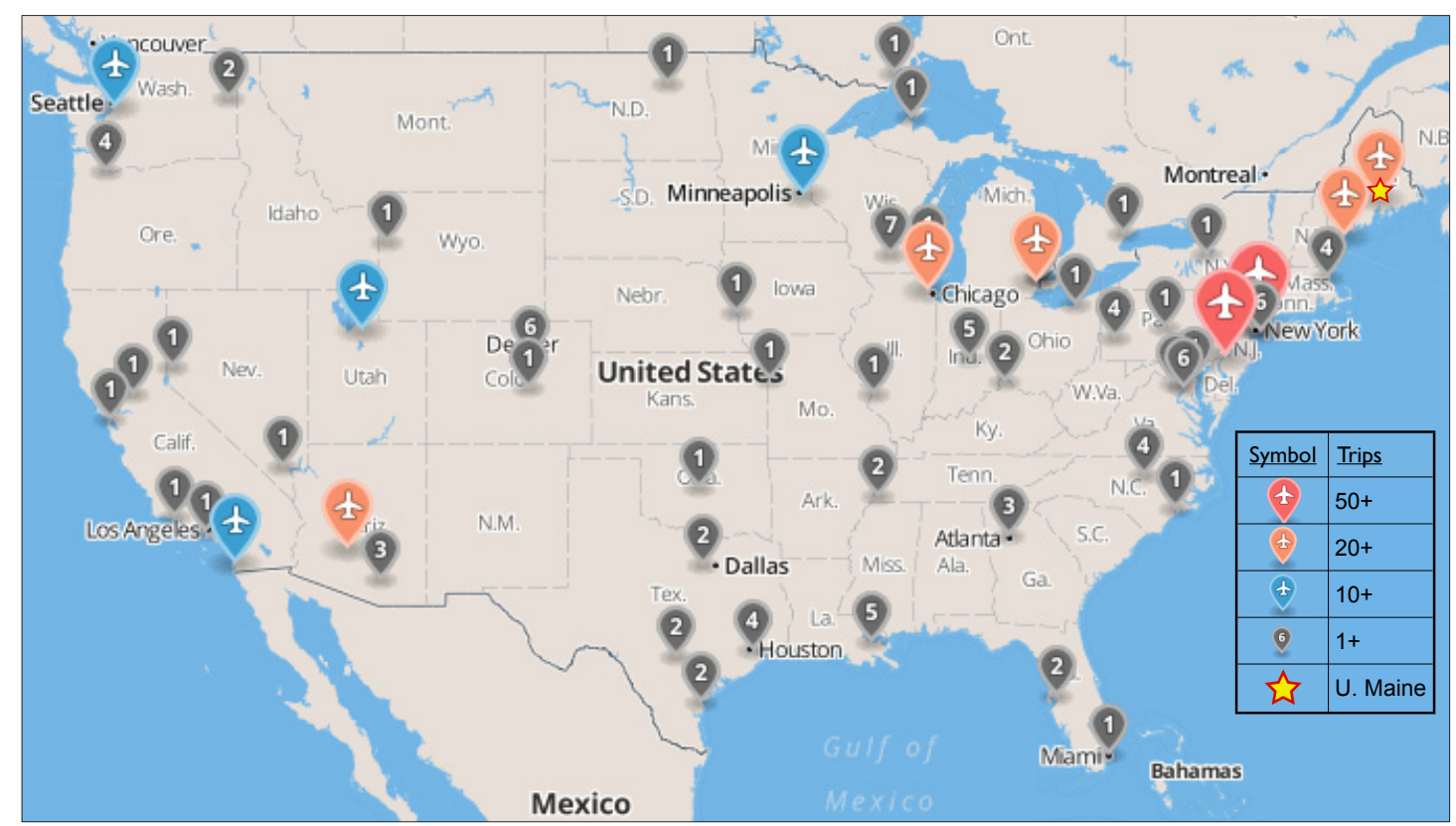

We used standard and reproducible methods to calculate the carbon intensity of each trip. Since the actual vehicle fuel efficiency was not known for specific trips, we used the U.S. Department of 
Transportation 2009 average fuel efficiency for "Light Duty Vehicles, short wheel base" [23], an efficiency of 23.8 miles per U.S. gallon. We also assumed $8.91 \mathrm{~kg}$ of $\mathrm{CO}_{2}$ emissions per U.S. gallon of gasoline burned [24]. This yields an average carbon travel efficiency of $0.196 \mathrm{kgCO}_{2} / \mathrm{km}$. For air travel, we used the International Civil Aviation Organization (ICAO) Carbon Emissions Calculator (version 5) to estimate emissions for each leg of air travel reported on the project. The ICAO Carbon Emissions Calculator comprises an 11-step calculation that combines the user input of destination and departure cities with the best available datasets on fuel $/ \mathrm{km}$ by airplane type, airplane load factors by global travel region, number of seats by aircraft type, cabin class, and great circle distances to compute $\mathrm{CO}_{2}$ emissions [25]. This method provides us with the best possible estimate of the actual carbon intensity of air travel. In addition, we performed an exploratory analysis of variance (ANOVA) summarized in the appendix.

\section{Results and Discussion}

Total carbon emissions for the first three years of the five-year research effort came to approximately 100 metric tons of carbon dioxide $\left(100.5 \mathrm{tCO}_{2}\right)$. Both automobile and airplane travel varied across the three years of the project for which we have emissions data (Table 1). Fiscal year 2011 saw much more air and car travel than did either 2010 or 2012 , with a total emission $\left(58.4 \mathrm{tCO}_{2}\right)$ of more that both prior and following years combined. Emissions from air travel were more than twice that of emissions from automobile travel. Trips by air averaged $691.7 \mathrm{kgCO}_{2}$, while the mean emissions per car trip were $101 \mathrm{kgCO}_{2}$.

Table 1. Estimated carbon emissions of research-related travel by fiscal year.

\begin{tabular}{|c|c|c|c|c|c|c|}
\hline Fiscal year & Auto trips & Auto miles & $\begin{array}{l}\text { Auto emissions } \\
\qquad\left(\mathrm{kg} \mathrm{CO} \mathrm{CO}_{2}\right)\end{array}$ & Air trips & $\begin{array}{c}\text { Air } \\
\text { emissions } \\
\left.(\mathrm{kg} \mathrm{CO})_{2}\right)\end{array}$ & $\begin{array}{c}\text { Total } \\
\text { emissions } \\
(\mathrm{kg} \mathrm{CO})\end{array}$ \\
\hline 2010 & 96 & 22,620 & 8468 & 23 & 14,030 & 22,499 \\
\hline 2011 & 140 & 40,635 & 15,200 & 67 & 43,271 & 58,484 \\
\hline 2012 & 67 & 18,624 & 6972 & 20 & 12,562 & 19,534 \\
\hline Total & 303 & 81,879 & 30,640 & 101 & 69,863 & 100,517 \\
\hline
\end{tabular}

Individual trips may include multiple air travel legs and as well as car travel. The average trip emission was $249.4 \mathrm{kgCO}_{2}$, while the greatest was $1618 \mathrm{kgCO}_{2}$. The 10 most emissions-intensive trips each generated more than one metric ton of carbon dioxide, while there were 60 trips that each created more than a half-ton $\left(500 \mathrm{kgCO}_{2}\right)$. Greater than $50 \%$ of all trips produced less than $250 \mathrm{kgCO}_{2}$ (Figure 2). The fact that the majority of trips generated small carbon emissions reflects the focus of the SSI on sustainability research within the state of Maine. The emphasis on in-state research and stakeholders is therefore likely to reduce total project emissions in comparison to an equivalent project with a regional, national or international mission.

Carbon emissions can also be considered by individual. The mean annual emissions per person was $558.6 \mathrm{kgCO}_{2}$, with a maximum of $2561 \mathrm{kgCO}_{2}$ and a minimum of $14 \mathrm{kgCO}_{2}$. Average yearly emissions per individual were thus very unevenly distributed, with a few high emitters, and a long tail comprised of many medium and low emitters (Figure 3). Fifteen individuals emitted more than $1 \mathrm{tCO}_{2}$ 
when averaged across the three years, only one emitted more than $2 \mathrm{tCO}_{2}$ and 58 researchers produced less than a half-ton per year on average. A similar left-skewed distribution is found with total individual emissions (see Appendix).

Figure 2. A histogram of trips by emission intensity shows that the majority of travel produced less than $250 \mathrm{kgCO}_{2}$, while the most carbon intensive trip produced $1618 \mathrm{kgCO}_{2}$.

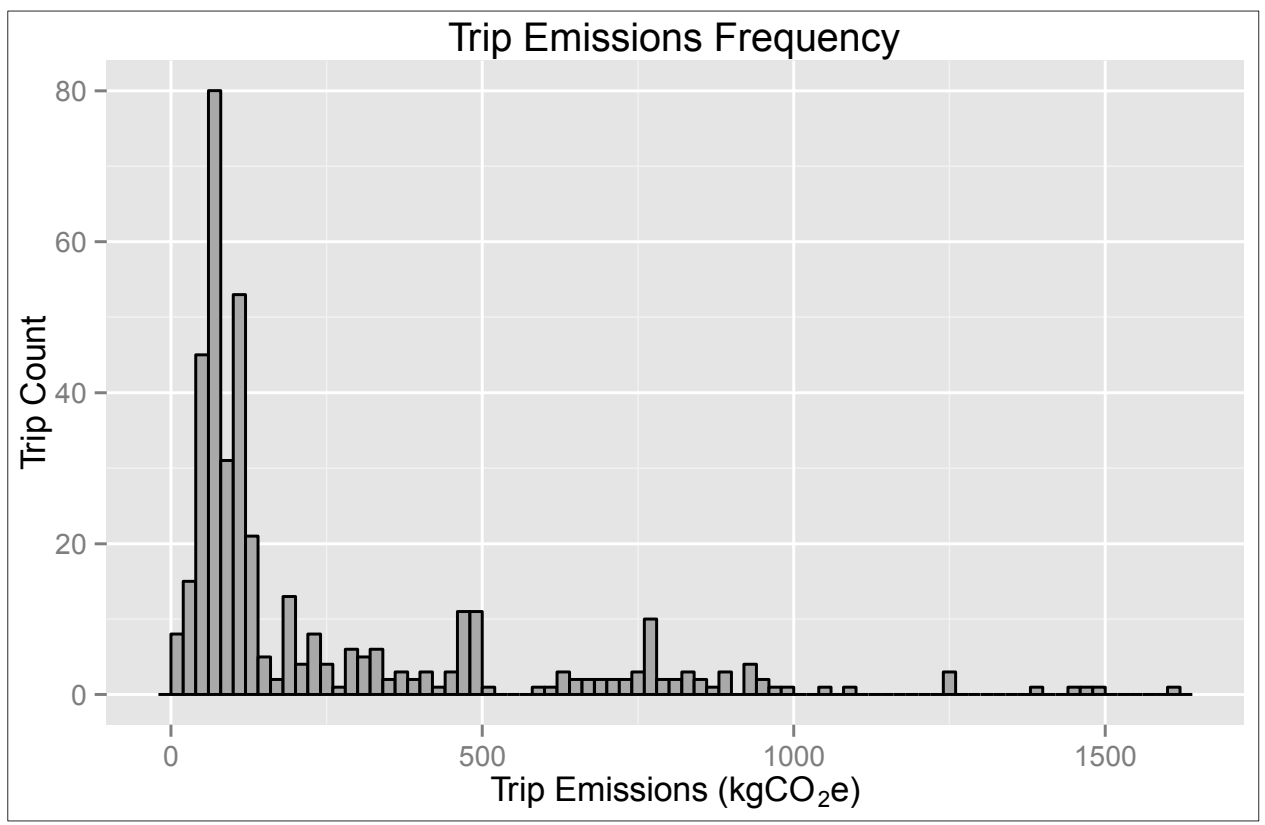

Figure 3. Annual average carbon emissions for sustainability research related travel by traveler, in descending order.

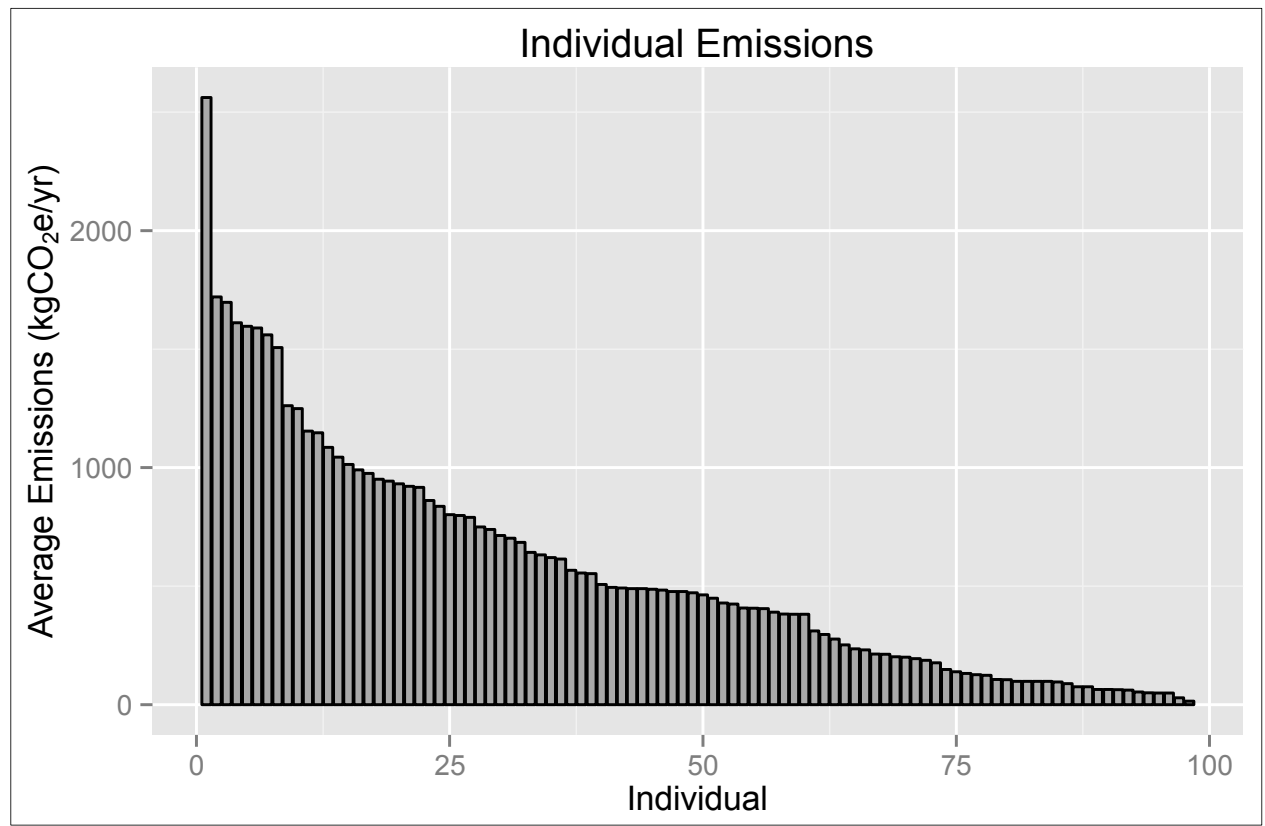

It is useful to assemble some context for our findings. A basic estimate of the carbon emissions of the steam plant that heats the majority of the University of Maine's campus is $27,000 \mathrm{tCO}_{2} /$ year. Spread across the population of $\sim 11,000$ students and faculty, this yields a per capita emissions rate of 
$2.5 \mathrm{tCO}_{2}$ /year - equivalent to the highest individual travel emissions in our study. Assuming that the highest emitter in our study is an unusually active researcher, we can conclude that the emissions cost of heating in Maine likely greater, on average, than the emissions costs of travel for most in the university community. Unfortunately, estimating the Scope 1 (from sources owned and controlled by the project) and Scope 2 (from electricity, heat purchased) emissions of the research project for comparison with or Scope 3 travel emissions estimate was not possible given the available data. Emissions were also tallied by disciplinary category, type of traveler, fiscal year. Data summaries for these variables are available in the appendix.

We also categorized travel data by the purpose of travel (coded from the travel expense vouchers). The purpose of travel had an important effect on trip length and carbon intensity. Travel for the purpose of dissemination (including presentations and conferences) tended to be to more distant locations, and thus emit more carbon than travel for administration, research or other purposes (Figure 4). However, aside from a cluster of in-state trips to in-state meetings, the majority of travel for the purpose of dissemination was out of state, and required extensive air travel. Thus, travel undertaken for the purpose of dissemination was more carbon intensive than research, research administration and other purposes (Figure 4).

Figure 4. Carbon emissions by purpose of travel.

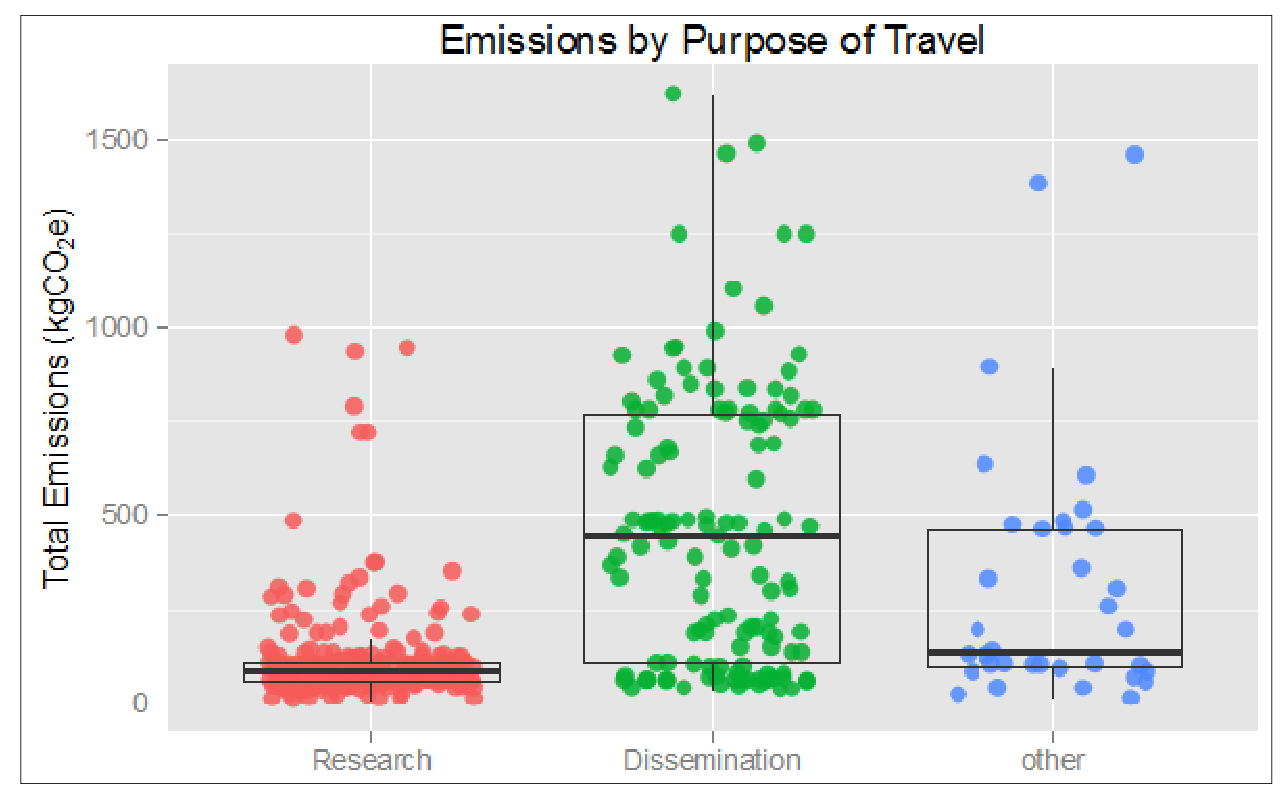

Notes: Each dot represents a single trip; points are jittered on the $\mathrm{x}$ axis; Research includes research administration travel; and Dissemination includes conference and presentation travel.

Further analysis, including an analysis of variance can be found in the appendix. The dataset is available at [26] and the $\mathrm{R}$ code used to analyze the data is also at [27].

\section{Conclusions}

For the purposes of travel emissions calculation, this analysis improves on the approaches of Fox et al. [17], and Ozawa-Meida et al. [22] in two ways. First, we are able to calculate emissions based on precisely documented travel rather than travel estimates based on expenditures. Combining 
this higher quality data with the ICAO version 5 emissions calculator gives our estimates high accuracy. Second, we were able to measure all of the travel of a large organization over a three-year time span. This gives us a better picture of the distribution of carbon emissions across the individuals and activities in an academic research setting.

One limitation of our methods is that they only capture a slice of an individual's travel emissions. The ideal measurement would be to normalize the emissions to the $\% \mathrm{FTE}$ (full time equivalent) of each researcher who contributed to the project, to produce an emissions/FTE measure. We were not able to accomplish in our study. It is reasonable, though, to assume that the individuals in this study have travel associated with other research or outreach projects as part of their professions, emissions from personal travel, and emissions from non-travel aspects of daily life in an industrialized society. It is also likely that the amount of personal travel individual researchers conduct, may respond to the addition of research travel in their professional lives. For instance, personal travel might decrease because individual tolerances for travel becomes somewhat saturated, or because such personal travel is joined with professional travel by piggybacking personal trips on research trips. Or, alternatively, personal travel might increase as researchers become more comfortable with higher amounts of travel, and willing to spend more money on travel. Our data do not allow us to evaluate these questions, but they do help us think more concretely about the tradeoffs of travel emissions by sustainability scientists.

The biggest limitation of travel emissions research in general is that the social benefits of the travel are themselves impossible to tally, or even estimate. For example, imagine that climate and sustainability researchers generate, through their research, education, policy advocacy, and outreach, a long-term net negative effect on carbon emissions. If that were the case their travel for research and dissemination supports this long-term emissions reduction. While this is possible, we think this is unlikely in most cases. Importantly, even if it were possible to measure these long-term benefits, by definition, they could only be measured once it is too late to influence policy that mattered at the time of travel. Therefore, the argument that as researchers, our travel warrants special consideration and exemption from collective efforts to reduce travel carbon emissions because of our special purpose seems misguided.

What, then, is the appropriate frame for the issue of greenhouse gas emissions by sustainability and climate change researchers and educators? How should we balance the tradeoff between the research enterprise and its environmental and social costs? As we have argued above, tallying environmental and economic costs and benefits does not appear very feasible. Moreover, we suspect that solutions derived from cost-benefit approach will tend to be marginal in effect. An alternative is to frame the issue as a problem of cooperation and collective action [28]. Global carbon emissions are themselves a collective action problem, and the emission of carbon by sustainability researchers is really the same problem, ramified by the demands of the academy.

Clearly transport, including individual air travel, is a significant causal factor in anthropogenic climate change [29]. Finding solutions to the sustainability challenge presented by climate change will require change at multiple dimensions, both individually and collectively. One way to address this collective action problem is to ask the question of how much greenhouse gas emission is too much for an individual, project, institution, nation, or the world. Since we have data here at the level of the individual, we looked for the answer to this question at the level of the individual. While there is broad consensus in the climate science community that overall greenhouse gas emissions need to be reduced 
to reduce the threat of climate change, the questions of how much reduction and who should reduce are not clear. One reasonable approach is that of Chakravarty et al. [30], who argue for a "cap" on individual emissions of $9.6 \mathrm{tCO}_{2}$ per year in order to achieve climate stabilization in the future and alleviate poverty by assuming the current distribution of emissions remains with the caveat that everyone on the planet needs at least $1 \mathrm{tCO}_{2}$ per year [30]. While they do not argue for a literal cap at this level, they suggest this "high emitters" cap is an equitable means of allocating emissions.

Assuming that about $10 \mathrm{tCO}_{2}$ per year is a reasonable upper limit on total emissions before an individual is contributing too much to climate change, we can begin to consider what our data might mean about the paradox of sustainability research related travel. Fifteen of the individuals in the study generated emissions of ten percent or more of this annual amount on this one research project alone. Over 40 individuals generated roughly 5 percent or more of an annual allocation on this project. One outlier had more than a quarter of this possible annual emissions cap. As Fox et al. [17] suggest for their group, the travel emissions of sustainability scientists in our study are likely to be greater than the U.S. national average and above a reasonable cap that would limit contributions to climate change in the future. Adding the per capita emissions due to heating at the University of Maine, as well as home heating, personal travel, and consumption rapidly approaches Chakravarty's cap.

From a policy perspective there are several possible responses to the individual travel behavior that drives these findings. One response is to address researcher emissions as an individual problem, and design policies that target reduction options and incentives toward those who emit more, such as a carbon tax. Some argue that they do or should purchase carbon offsets for their professional travel [18]. But because many do not have such means the offset response appears more like a band-aid than a solution. Thankfully, potential solutions to the conflict between the individual travel needs of sustainability researchers and the global need for climate stabilization abound. They include reporting, technological alternatives to travel, incentive and institutional change, and innovation in the realm of social contracts.

Our research shows that recording and reporting of travel emissions in large research ventures is feasible, and we argue that doing so is a keystone of any effective emissions reduction policy. We recommend that research-related travel emissions become a central part of the conversation among sustainability scientists. To achieve this, large scale reporting is needed, as exemplified by the efforts of the Higher Education Funding Council for England [11]. Research institutions and universities should maintain travel emissions records at both the project and individual levels as part of larger institutional footprinting efforts [22]. Individual scientists should keep such records for themselves and understand how their professional emissions compare with other scientists and with their own emissions in their personal lives [31]. But recording and reporting is only a preliminary step.

Teleconferencing technology can reduce the need for travel by enabling research collaboration and presentation across vast distances. Modern teleconferencing technology is cheap and high quality. However, for many research activities such as data collection there is no alternative, so these technologies can only reduce travel to a point. Moreover, it is unknown how these technologies interact with travel over time. Research is needed to determine if telecommunications technologies function as effective replacements for travel or as complements or catalysts for additional travel with new collaborators. There is anecdotal evidence of both effects. On the one hand, experts will increasingly give guest lectures and keynote addresses via Skype [32]. This could either act as a 
substitute for travel, reducing emissions, or facilitate busier schedules with more travel, by making long distance collaborations more stable and productive, leading naturally to more visits. This would be good for research, but bad for travel emissions. In the SSI, teleconferencing was used extensively, mostly to facilitate in-state collaborations. This added distance communication increased collaboration between remote sites, but it is unclear if the effect of that increased collaboration on physical travel was positive or negative.

One domain in which teleconferencing technologies are still lacking is in effectively facilitating large group interactions. But, even in this domain, progress can be seen. For example, author T.W. recently participated in a nation-wide virtual poster session hosted on a National Science Foundation website (IGERT.org). Despite the challenges of using a website for collective interaction, the poster session was very effective and engaging. With the growing industry of online education, massive open online courses (MOOCs), and the proliferation of free videoconferencing technologies, the barriers to substituting virtual interaction for face-to-face interaction are falling rapidly. It remains an open question, however, how much and how well these distributed and technologically enabled interactions can truly substitute for real human gatherings.

If behavior is to change in the short or medium term, emissions must trigger social or economic costs, which can help drive behavioral adoption. An institutional change in individual incentives is the broadest strategy. For example if research-funding agencies such as the National Science Foundation, the National Institutes of Health, Department of Energy, United States Department of Agriculture and others attached stipulations and limits to travel on funded research, improvements could come very quickly. Universities themselves could implement research-greening policies related to research travel. Similarly, institutions of national and international oversight, accreditation and ratings bodies such as the Association for the Advancement of Sustainability in Higher Education (AASHE) and the American College and University Presidents' Climate Commitment (APUPCC) could influence major change. These voluntary organizations have emerged in order to create a long-term structure for positive change in university operations, and are naturally positioned to push for travel-related changes in research activity. Institutional change also has drawbacks as a top-down solution, and sometimes bottom-up solutions are more effective at capturing individual focus.

The institutions and traditions of academia also place a premium on the prestige of individual researchers, requiring travel in the name of professional advancement, conference presentations and collaboration. Our most prominent finding (in Figure 4) is that conference attendance generated some of the highest per trip emissions. Conference travel represents a major driver of total research-travel emissions, suggesting that academic conferences should be a first-round policy target. When the professional success of individual researchers, graduate students, and pre-tenure faculty rests in large part on their performance in the academic societies of their fields, we should expect individuals to be recalcitrant in voluntarily reducing their research and conference emissions. A bottom up strategy is therefore unlikely to emerge without significant institutional change. Though it is tempting to try to find the institution through which policy change would have the greatest effect, we suspect there is no such single answer. Instead, the responsibility falls on individuals, universities, professional societies, and funding agencies alike.

Thus, the collective carbon emissions of sustainability research appears to be a collective action problem. Collective action problems are overcome through changes in the fundamental social contract. 
The AASHE and APUPCC are engaged in attempting to influence the social contract of higher education institutions by altering the criteria by which they are compared. Likewise, sustainability researchers themselves should attempt to alter the social contract directly through public professional agreements to reduce and eventually eliminate non-essential research travel [19]. It seems likely that the social and political determination to implement these solutions will remain lacking until there is more prestige to be had in opting out than in digging in.

In conclusion, we suggest that each of these avenues should be pursued simultaneously. With changes in incentives, funding, institutional reporting requirements, and individual leadership, social contracts around travel in academia can change, and research and dissemination emissions can be reduced. We have presented an example of the first step of such a process; recording travel and reporting those emissions.

\section{Acknowledgments}

This research was conducted as part of Maine's Sustainability Solutions Initiative, supported by National Science Foundation award EPS-0904155 to Maine EPSCoR at the University of Maine and the Maine Agricultural and Forest Experiment Station. We thank Carol Hamel for her assistance in accessing records and two anonymous reviewers for guidance in developing this paper.

\section{Author Contributions}

M.A. developed the research concept, organized and oversaw the data collection effort, and wrote the first draft. E.M. analyzed the data with SAS and produced the ANOVA. M.T. contributed to the text and analysis process through guidance. T.W. edited the paper and analyzed the data using R.

\section{Appendix}

Here we provide additional data summaries and an analysis of variance for those interested in further exploring the emissions data.

Figure 5. Average monthly carbon emissions.

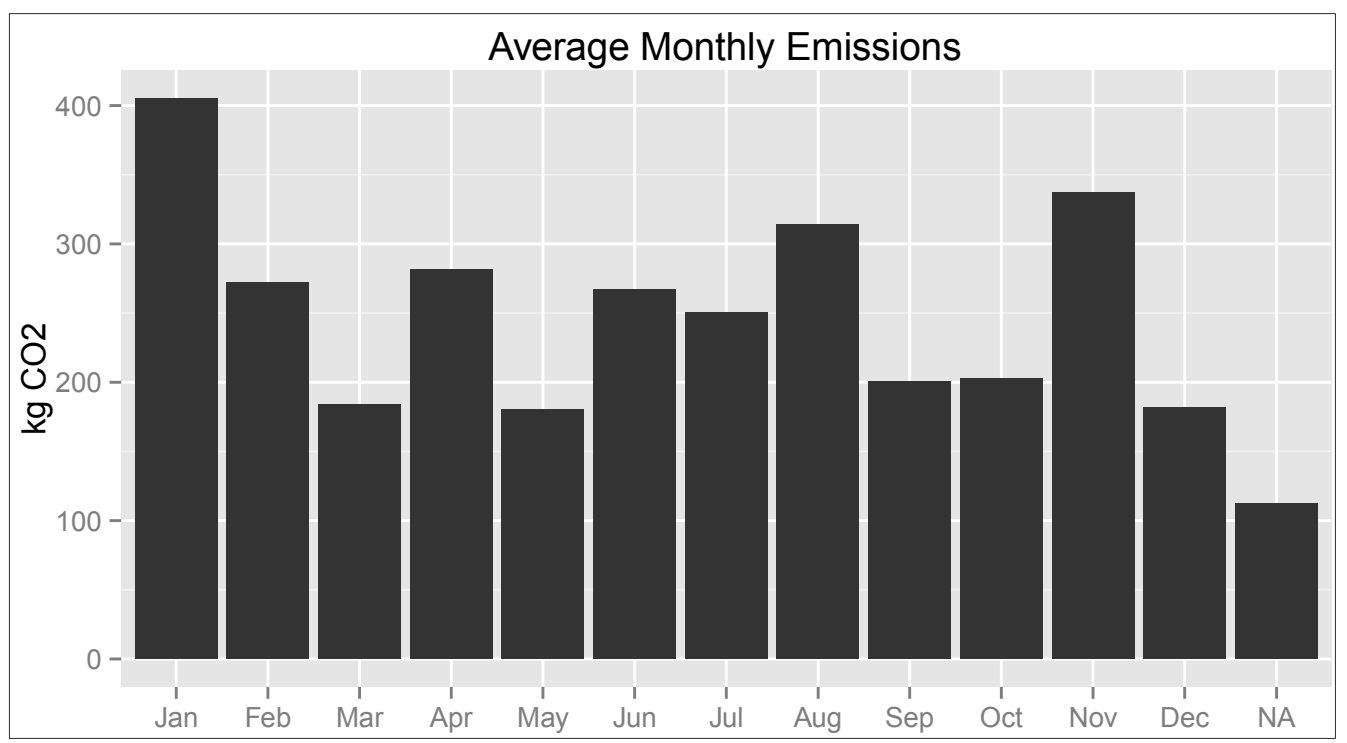


Figure 6. Total emissions across the three fiscal years by individual, ordered by emissions.

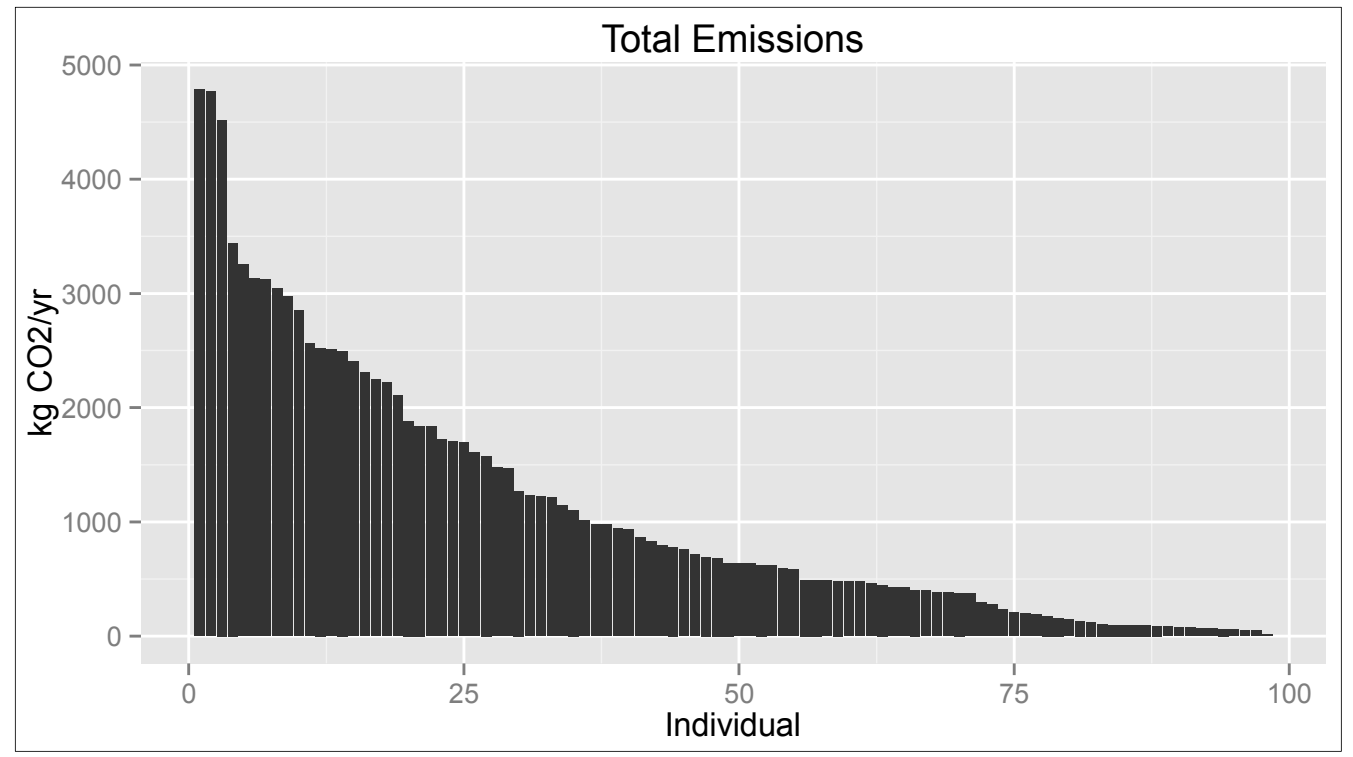

Figure 7. Carbon emissions per trip by academic season.

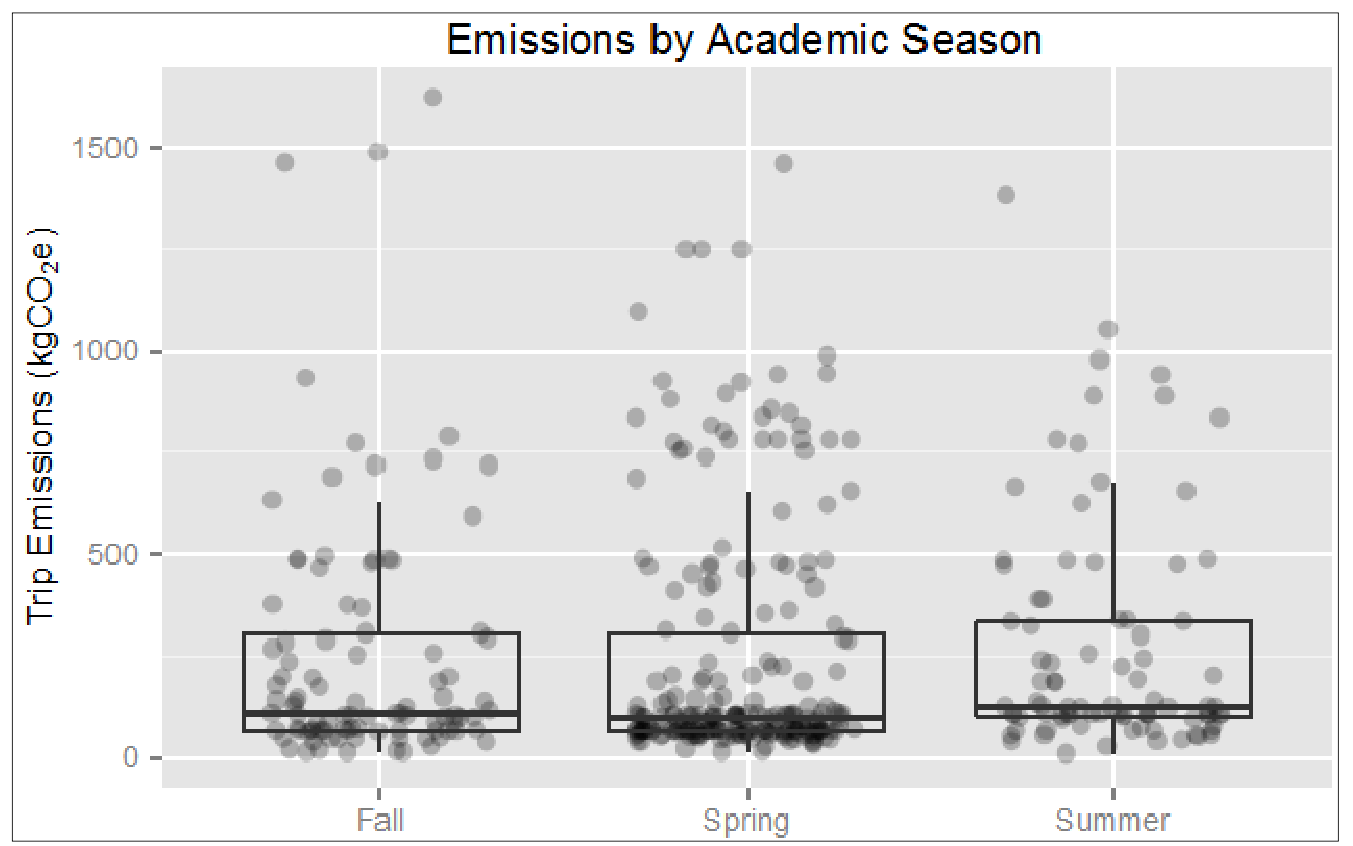

Notes: Spring includes January through May; Summer includes June through August; Fall includes the remainder of the year. 
Figure 8. Carbon emissions per trip across the fiscal years covered within the dataset.

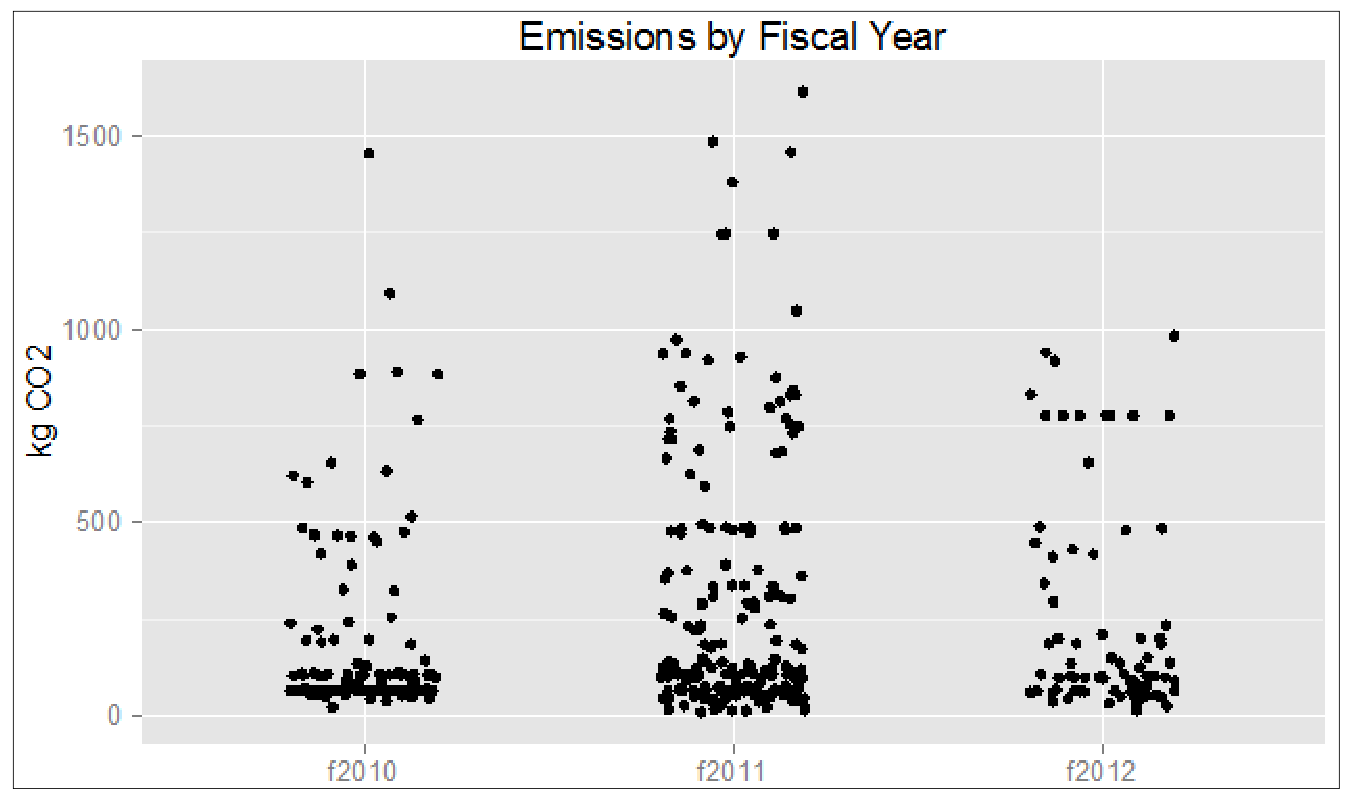

Note: Each point represents one trip.

Figure 9. Total travel emissions by calendar year.

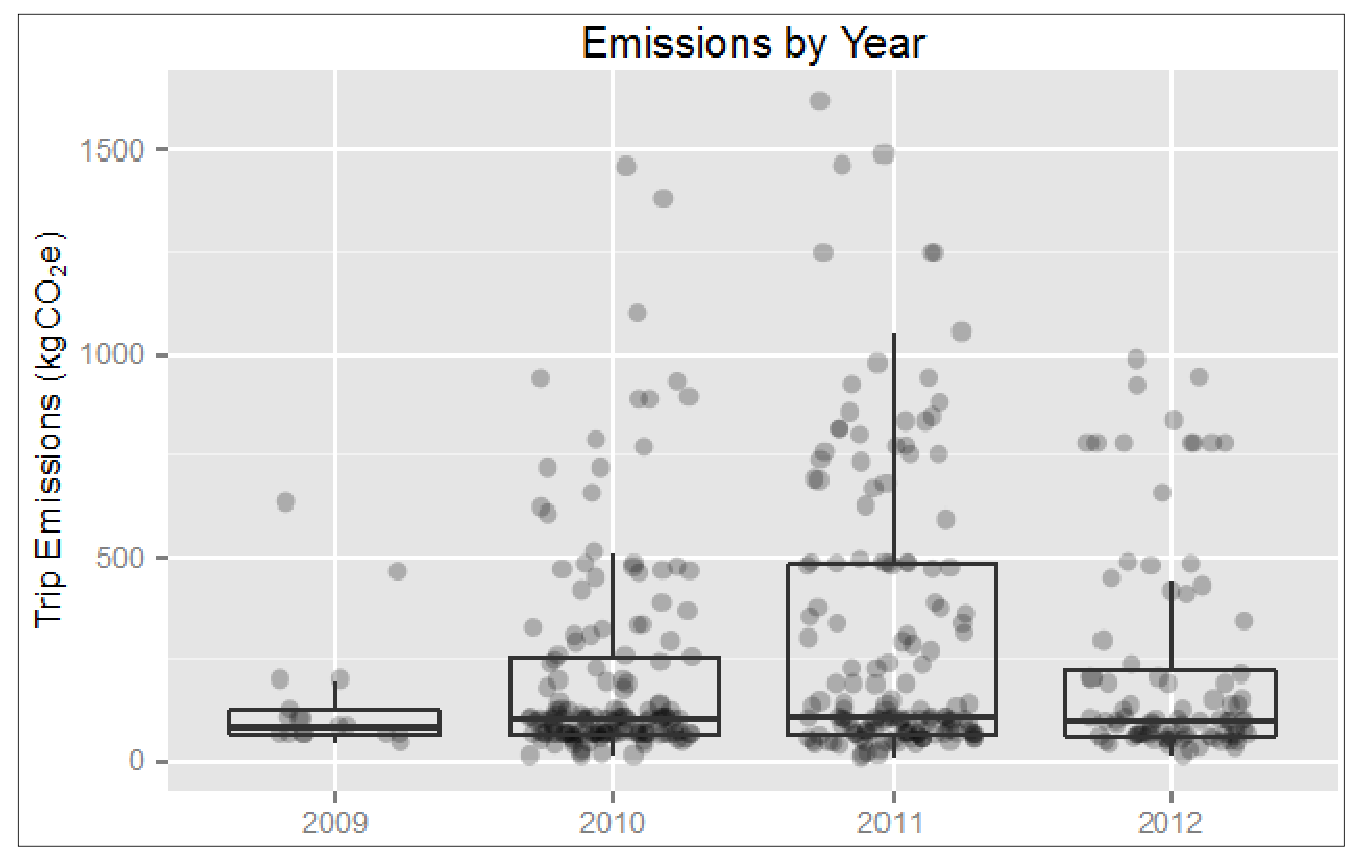


Figure 10. Carbon emissions frequency by purpose of travel.

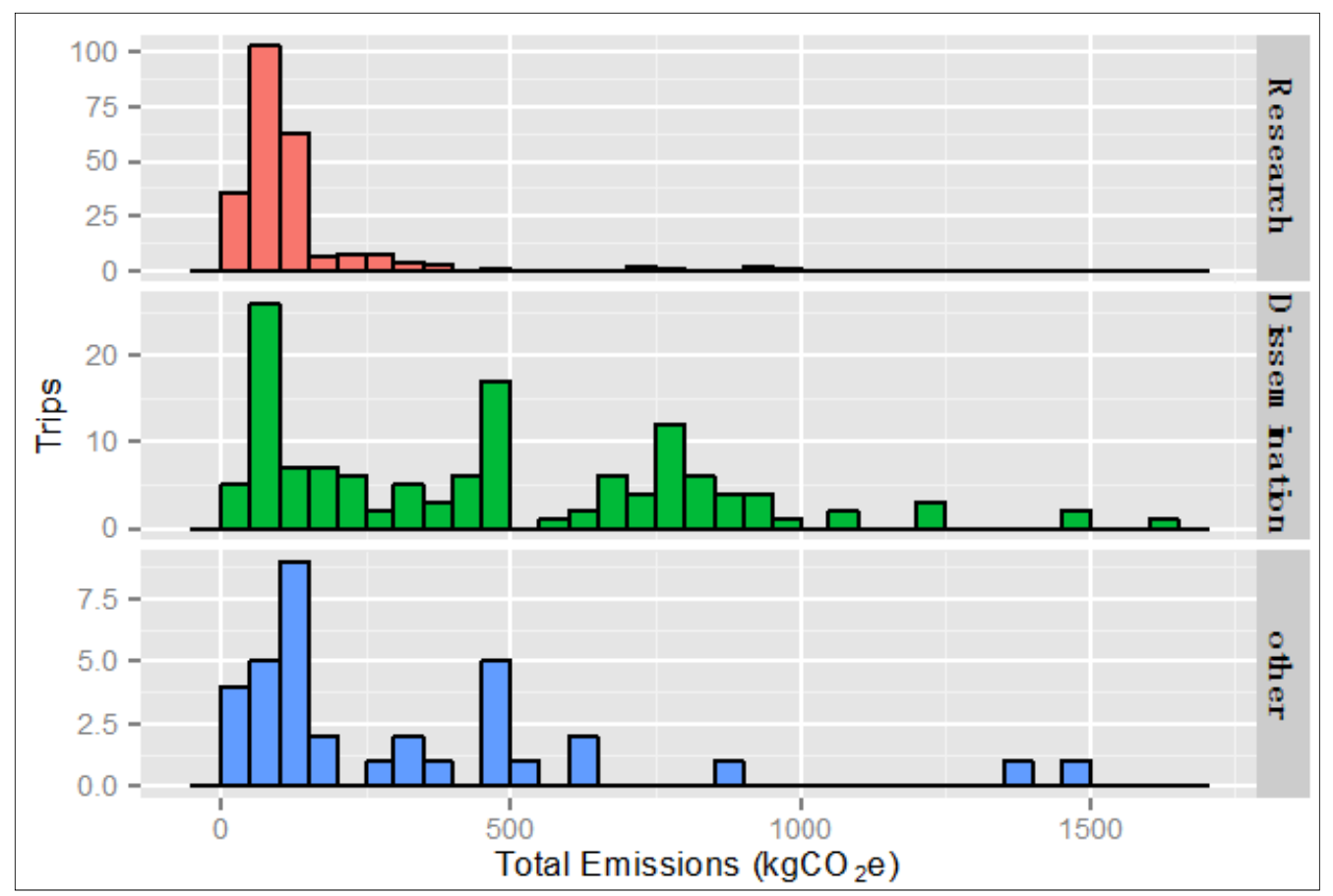

Histograms in Figure 10 reveal that the bulk of research travel (research and administration) falls below the $500 \mathrm{~kg}$ mark, while approximately half of the dissemination travel (conferences and presentations) is above $500 \mathrm{~kg} \mathrm{CO} /$ trip.

Figure 11. Annual average carbon emissions by traveler type.

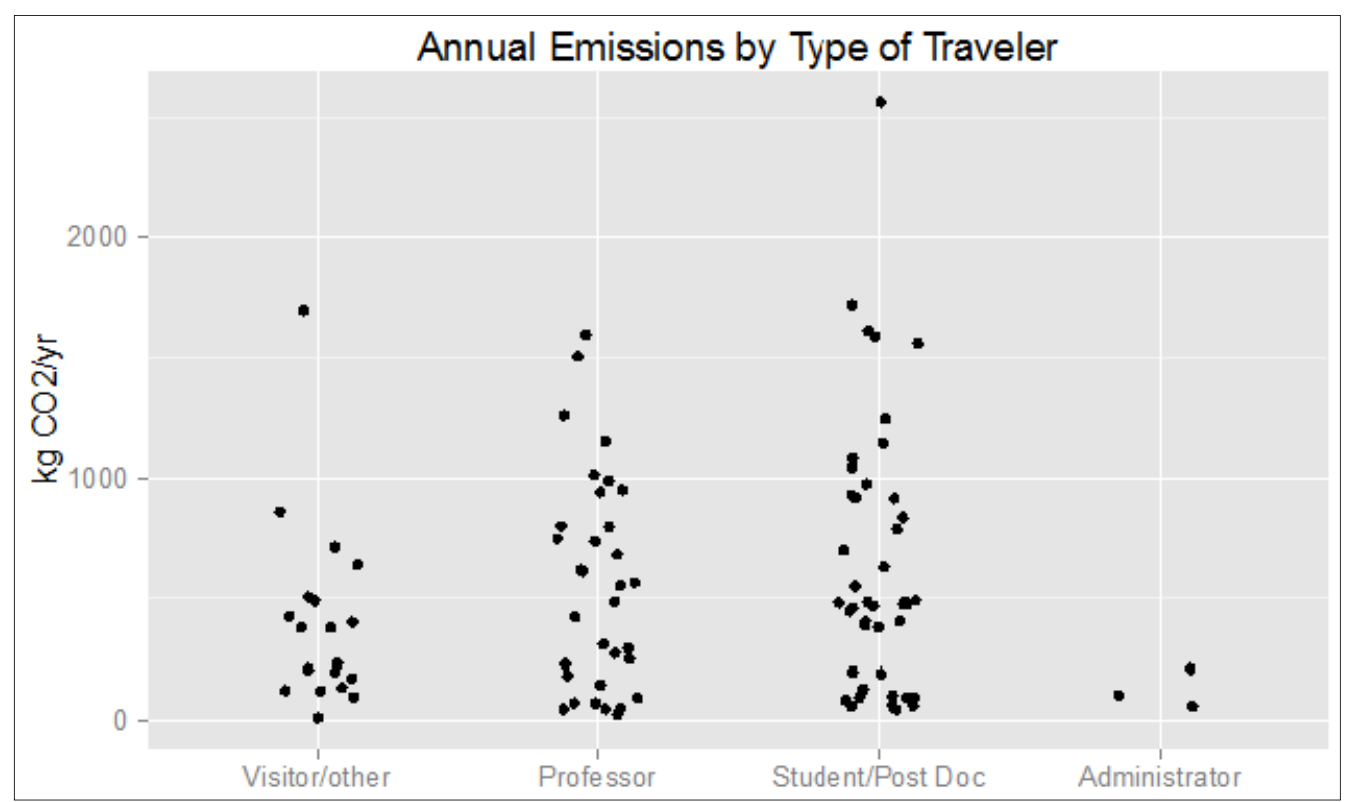

Note: Each dot represents one person and points jittered on the $\mathrm{x}$ axis. 
Figure 12. Boxplots of annual average carbon emissions due to travel by disciplinary super-category (natural science, social science, other).

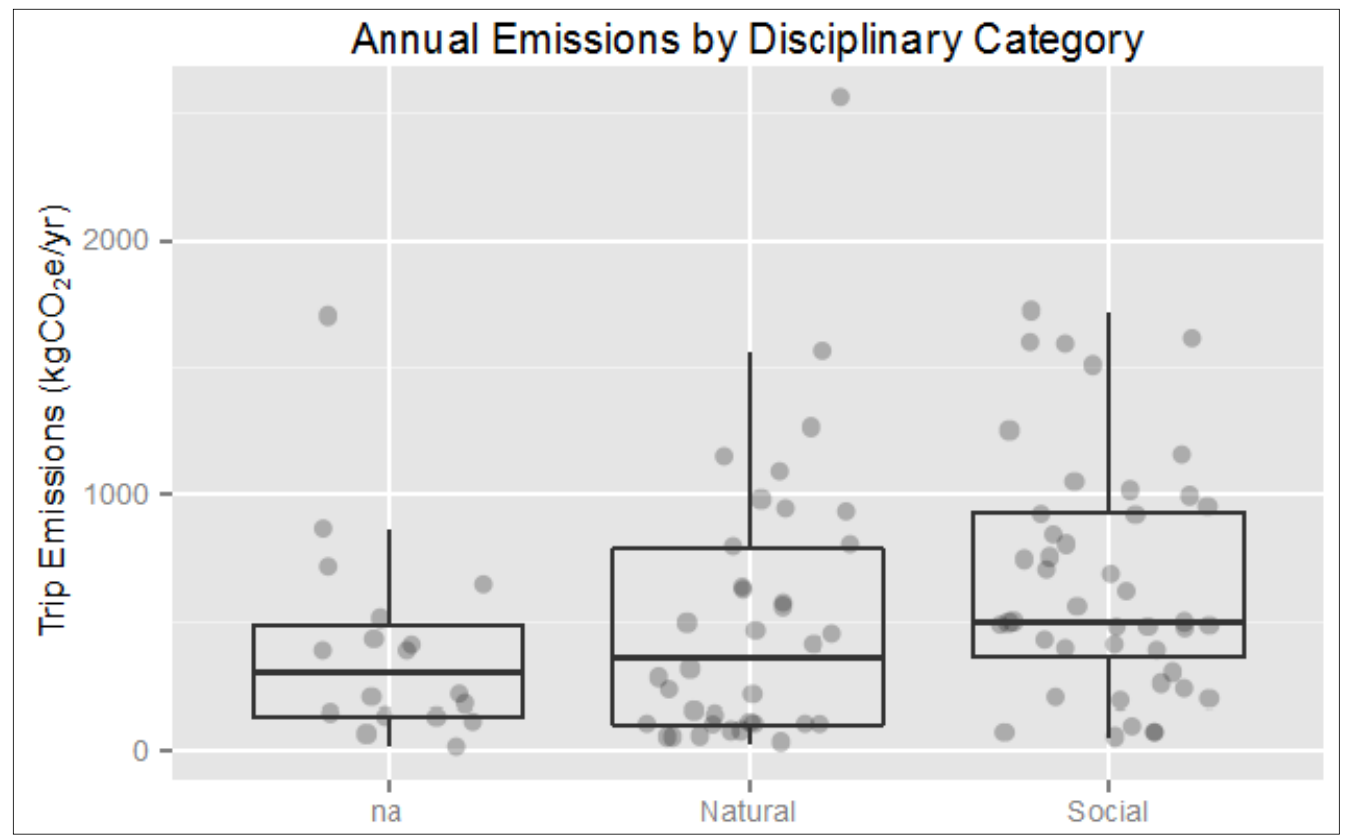

Table 2. Travel expenditure across the five years of the project comes to $1.2 \%$ of the total \$20 million budget.

\begin{tabular}{|c|c|c|c|c|c|c|c|c|}
\hline \multirow{2}{*}{ Year } & \multicolumn{2}{|c|}{ In-State } & \multicolumn{2}{|c|}{ Out-of-State } & \multicolumn{2}{|c|}{ International } & \multicolumn{2}{|c|}{ Total } \\
\hline & NSF & MEIF & NSF & MEIF & NSF & MEIF & NSF & MEIF \\
\hline 1 & 9371 & 1513 & 27,572 & 410 & - & 6438 & 36,943 & 8361 \\
\hline 2 & 9605 & 1485 & 48,665 & 4146 & - & - & 58,270 & 5631 \\
\hline 3 & 4718 & 11,039 & 25,765 & 61,681 & - & - & 30,483 & 72,720 \\
\hline 4 & 11,699 & 2979 & 47,972 & 12,362 & - & - & 59,671 & 15,341 \\
\hline 5 & 6179 & 2157 & 44,736 & 21,721 & - & - & 50,915 & 23,878 \\
\hline
\end{tabular}

(1) Analysis of Variance

Additionally, we conducted an analysis of variance of carbon emission on basic variables including year, month, travel type, traveler type, and discipline (Table 3). This ANOVA is presented for the purposes of data exploration because we do not hold any a priori hypotheses.

Table 3. Analysis of variance for carbon emissions using disaggregated data, calculated in SAS.

\begin{tabular}{cccc}
\hline Variable & Estimate & Standard error & Significance \\
\hline Intercept & 71.413 & $(105.036)$ & \\
Year 2009 & -22.875 & $(83.225)$ & \\
Year 2010 & 25.959 & $(74.762)$ & \\
Year 2011 & 83.166 & $(37.862)$ & $*$ \\
January & 180.495 & $(74.762)$ & $* * *$ \\
February & 32.330 & $(74.168)$ & \\
March & -83.385 & $(67.443)$ & \\
April & 30.491 & $(69.714)$ & \\
\hline
\end{tabular}


Table 3. Cont.

\begin{tabular}{cccc}
\hline Variable & Estimate & Standard error & Significance \\
\hline May & 11.994 & $(64.452)$ & \\
June & 25.195 & $(67.232)$ & \\
July & 42.551 & $(73.298)$ & \\
August & 99.660 & $(86.434)$ & \\
September & 10.241 & $(88.870)$ & \\
October & -39.755 & $(71.706)$ & \\
November & 94.7184 & $(67.406)$ & \\
Visitor & 140.660 & $(88.821)$ & \\
Full Professor & 124.369 & $(75.180)$ & $* *$ \\
Associate Professor & 382.626 & $(93.082)$ & $* * *$ \\
Assistant Professor & 176.518 & $(73.807)$ & $* * *$ \\
Post Doc. & 470.937 & $103.494)$ & $* * *$ \\
Ph.D. & 191.900 & $(73.211)$ & $* * *$ \\
MS & 212.117 & $(80.437)$ & $* * *$ \\
Research & -163.363 & $(63.781)$ & $* *$ \\
Conference & 145.242 & $(52.178)$ & $* * *$ \\
Administrative & -188.476 & $(48.875)$ & $* * *$ \\
Social Science & -18.304 & & \\
R squared & 0.4039 & $(29.423)$ & \\
Adj R-Sq & 0.3643 & & \\
No. Observations & 403 & & \\
\hline
\end{tabular}

Notes: $*, * *, * *$ indicates significance at the $90 \%, 95 \%$, and $99 \%$ level, respectively; intercept values include year $=2009$, month $=$ December, Traveler Type $=$ Administrative Staff, Travel Type $=$ Other, Disciplinary Category $=$ Natural.

(2) Data Availability

The data used in this study are available at http://dx.doi.org/10.7910/DVN/25532. With the exception of the ANOVA, which was computed in SAS, annotated R code used to process, analyze and visualize the data is available at http://rpubs.com/twaring/ssi_carbon.

\section{Conflicts of Interest}

The authors declare no conflict of interest.

\section{References and Notes}

1. Intergovernmental Panel on Climate Change (IPCC). IPCC Fifth Assessment Report; IPCC Secretariat, World Meteorological Organization: Geneva, Switzerland, 2014.

2. Barrett, S. The Incredible Economics of Geoengineering. Environ. Resour. Econ. 2008, 39, 45-54.

3. Corner, A.; Pidgeon, N. Geoengineering the climate: The social and ethical implications. Environ. Sci. Policy Sustain. Dev. 2010, 52, 24-37.

4. Robock, A. 20 reasons why geoengineering may be a bad idea. Bull. At. Sci. 2008, 64, 14-18. 
5. Gowdy, J.M. Behavioral economics and climate change policy. J. Econ. Behav. Organ. 2008, 68, 632-644.

6. Dietz, T.; Gardner, G.T.; Gilligan, J.; Stern, P.C.; Vandenbergh, M.P. Household actions can provide a behavioral wedge to rapidly reduce US carbon emissions. Proc. Natl. Acad. Sci. 2009, 106, 18452-18456.

7. Levin, S.A.; Clark, W.C. Toward a Science of Sustainability; CID Working Paper No. 196; The National Science Foundation: Warrenton, VA, USA, 2010; p. 109.

8. Al Gore Cartoon. Fellowship of the Minds. Available online: http:/fellowshipofminds. files.wordpress.com/2011/05/al-gore-cartoon.jpg (accessed on 7 April 2014).

9. Corporate Standard. Greenhouse Gas Protocol. Available online: http://www.ghgprotocol.org/ standards/corporate-standard (accessed on 7 April 2014).

10. International Standards Organization (ISO). ISO 14064-1:2006-Greenhouse gases-Part 1: Specification with guidance at the organization level for quantification and reporting of greenhouse gas emissions and removals. Available online: http://www.iso.org/iso/catalogue detail?csnumber=38381 (accessed on 7 April 2014).

11. Higher Education Funding Council for England (HEFCE). Carbon Reduction Target and Strategy for Higher Education in England; Higher Education Funding Council for England: Bristol, UK, 2010. Available online: https://www.hefce.ac.uk/pubs/year/2010/201001/ (accessed on 8 April 2014).

12. Association for the Advancement of Sustainability in Higher Education (AASHE). Available online: https://stars.aashe.org/ (accessed on 7 April 2014).

13. Second Nature. Education for Sustainability. Available online: http://www.secondnature.org/ (accessed on 7 April 2014).

14. American College and University Presidents' Climate Commitment (APUPCC). Instructions for Submitting a Greenhouse Gas Report. Available online: http://rs.acupcc.org/instructions/ghg/ (accessed on 7 April 2014).

15. Michaelowa, A.; Lehmkuhl, D. Greenhouse gas emissions caused by the international climate negotiations. Clim. Policy 2004, 4, 337-340.

16. Grémillet, D. Paradox of flying to meetings to protect the environment. Nature 2008, 455, 1175.

17. Fox, H.E.; Kareiva, P.; Silliman, B.; Hitt, J.; Lytle, D.A.; Halpern, B.S.; Hawkes, C.V.; Lawler, J.; Neel, M.; Olden, J.D.; et al. Why do we fly? Ecologists' sins of emission. Front. Ecol. Environ. 2009, 7, 294-296.

18. Bossdorf, O.; Parepa, M.; Fischer, M. Climate-neutral ecology conferences: Just do it! Trends Ecol. Evol. 2010, 25, 61.

19. Anderson, K. Hypocrites in the air: Should climate change academics lead by example? Comment on Climate. Available online: http://kevinanderson.info/blog/hypocrites-in-the-air-should-climatechange-academics-lead-by-example/ (accessed on 4 December 2013).

20. Anderson, K.; Calverley, D.; Sharmina, M. Evangelising from 32 thousand feet: Why Brendan May is wrong in calling for more environmentalists to fly. Comment on Climate. Available online: http://kevinanderson.info/blog/evangelising-from-32-thousand-feet-why-brendan-may-iswrong-in-calling-for-environmentalists-to-fly-more/ (accessed on 7 April 2014). 
21. May, B. Why more environmentalists should fly. The Guardian. Available online: http://www.theguardian.com/environment/2013/nov/05/why-more-environmentalists-should-fly (accessed on 7 April 2014).

22. Ozawa-Meida, L.; Brockway, P.; Letten, K.; Davies, J.; Fleming, P. Measuring carbon performance in a UK University through a consumption-based carbon footprint: De Montfort University case study. J. Clean. Prod. 2013, 56, 185-198.

23. U.S. Department of Transportation (DOT), Bureau of Transportation Statistics. Average Fuel Efficiency of U.S. Light Duty Vehicles. Available online: http:/www.rita.dot.gov/bts/sites/ rita.dot.gov.bts/files/publications/national_transportation_statistics/html/table_04_23.html (accessed on 8 April 2014).

24. U.S. Energy Information Agency (EIA). Voluntary Reporting of Greenhouse Gases Program. Available online: http://www.eia.gov/oiaf/1605/coefficients.html (accessed on 16 June 2013).

25. International Civil Aviation Organization (ICAO). Carbon Emissions Calculator. Available online: http://www.icao.int/environmental-protection/CarbonOffset/Pages/default.aspx (accessed on 4 December 2013).

26. Waring, T. SSI Carbon Study. Available online: http://dx.doi.org/10.7910/DVN/25532 (accessed on 20 April 2014).

27. Waring, T. SSI Carbon Emissions Study. . Available online: http://rpubs.com/twaring/ssi_carbon (accessed Apr 20, 2014).

28. Olson, M. The Logic of Collective Action: Public Goods and the Theory of Groups; Harvard University Press: Cambridge, MA, USA, 1965.

29. Chapman, L. Transport and climate change: A review. J. Transp. Geogr. 2007, 15, 354-367.

30. Chakravarty, S.; Chikkatur, A.; de Coninck, H.; Pacala, S.; Socolow, R.; Tavoni, M. Sharing global $\mathrm{CO}_{2}$ emission reductions among one billion high emitters. Proc. Natl. Acad. Sci. 2009, 106, 11884-11888.

31. Anderson, M. Reducing Your Footprint: A Handbook for Reducing Household Carbon Dioxide Emission; School of Economics Staff Paper \# 567; University of Maine: Orono, Maine, USA; 2007.

32. Alaska Society for Technology in Education (ASTE). 2014 Keynote Speakers. Available online: http://www.aste.org/conference/speakers/ (accessed on 7 April 2014).

(C) 2014 by the authors; licensee MDPI, Basel, Switzerland. This article is an open access article distributed under the terms and conditions of the Creative Commons Attribution license (http://creativecommons.org/licenses/by/3.0/). 